\title{
Correction to: Towers of Hanoi problems: Deriving iterative solutions by program transformations
}

\author{
Alberto Pettorossi $^{1}$ \\ Published online: 28 November 2019 \\ (C) Springer Nature B.V. 2019
}

\section{Correction to: BIT 25 (1985), 327-334 \\ https://doi.org/10.1007/BF01934378}

Dear readers,

In the originally published version, the author found some subsequent corrections.

page 328 line -13 . The line between the two dots should connect them.

page 332 line +19 . (yy...y] should be [yy...y]

page 333 line +1 . sisks should be disks

page 333 line +2 . seuqnce should be sequence

page 333 line -18. :- should be :=

page 333 line -16 . res $:=\operatorname{hd}(1)::$ should be res $:=\operatorname{hd}(1) \mathrm{y}::$

page 333 line -9. $\operatorname{tl}(1)$, should be $\operatorname{tl}(1))$,

We apologize for these errors.

Publisher's Note Springer Nature remains neutral with regard to jurisdictional claims in published maps and institutional affiliations.

The original article can be found online at https://doi.org/10.1007/BF01934378.

Alberto Pettorossi

alberto.pettorossi@iasi.cnr.it

1 Istituto di Analisi dei Sistemi ed Informatica, CNR, Via dei Taurini 19, 00185 Rome, Italy 